\title{
Endoscopy as a Diagnostic Tool for Personalized Therapy in IBD: Prediction of Response to Biological Therapy
}

Both Crohn's disease [1] and ulcerative colitis [2] are chronic relapsing disorders of the gastrointestinal tract that are characterized pathologically by intestinal inflammation. Both inflammatory bowel disease (IBD) entities are progressive conditions that can lead to bowel damage and disability, having a major impact on an individual's quality of life. Furthermore, ongoing inflammatory activity is causative for occurrence of strictures, fistula, abscesses, as well as heightened incidence of colitisassociated neoplasia. Optimized anti-inflammatory therapy is therefore essential in the management of IBD patients [1,2]. Advances in understanding the underlying immunopathogenesis in ulcerative colitis have led to the development of biological therapies, which selectively inhibit crucial pathways of involved inflammatory processes. Blockade of the pro-inflammatory cytokine tumor necrosis factor (TNF) by neutralizing antibodies was the first approved treatment modality, which was complemented by the anti-adhesion antibody vedolizumab directed against the $\alpha 4 \beta 7$ integrin. Subsequently, the antiIL-12/IL-23p40 antibody ustekinumab and the JAK-inhibitor tofacitinib have broadened our therapeutic armamentarium. In spite of their effectiveness, it was found that only subgroups of IBD patients benefit from these therapies. Depending on the duration and the clinical endpoints chosen, only $30-60 \%$ of patients will show an improvement of active disease. There is therefore the currently unmet clinical need to predict therapeutic responses prior to the initiation of therapy. Reliable prediction would enable the treating physician to directly introduce the patient to the most efficient therapy, which would enable time-efficient control of the disease and prevent the onset of irreversible structural damage. This would also be essential to avoid exposure of patients to associated potential side effects of an inefficient therapy and reduce the substantial costs of an inappropriate therapy for the health care systems [3].

Here, a novel approach for the analysis of molecular signaling pathways in IBD patients by using labeled antibodies and con- focal laser endomicroscopy (CLE) for in vivo molecular endoscopy has lately been developed [4].

Preclinical experimental studies revealed that TNF antibodies induce T cell apoptosis in IBD by binding to membrane-bound TNF (mTNF) expressing mucosal cells and thereby blocking the mTNF/TNFR2 costimulation pathway between CD14+ macrophages and CD4+ T cells in the lamina propria [5]. Based on these findings, the anti-TNF antibody adalimumab was labeled with the fluorescein derivate fluorescein isothiocyanate (FITC) under GMP criteria. The fluorescent labeled anti-TNF antibody was then sprayed on the surface of the mucosa in Crohn's disease patients during ongoing CLE. This was the first clinical trial in which a GMP-conform, fluorescent antibody was used in humans. Topical antibody administration of the fluorescent anti-TNF antibody in 25 patients with Crohn's disease led to strong and specific signal detection of intestinal mTNF+ immune cells in vivo during CLE. Moreover, there was a difference regarding mTNF expression between the Crohn's disease patients in the study. Patients with high amounts of mTNF+ cells showed significantly higher short-term response rates at week 12 (92\%) upon subsequent anti-TNF antibody therapy as compared to patients with low amounts of mTNF+ cells (15\%). This clinical response in the former patients was sustained over a follow-up period of one year and associated with mucosal healing on follow-up endoscopy. No severe side effects or adverse events were noted [6]. Taken together, the findings of this clinical phase Il a study suggested that in vivo molecular endoscopy with fluorescent antibodies may allow the prediction of a therapeutic response to subsequent anti-TNF therapy.

A subsequent pilot study in patients with Crohn's disease demonstrated a correlation between the therapeutic efficacy of vedolizumab and the number of $\alpha 4 \beta 7$-expressing cells in the mucosa, which were detected using ex vivo CLE with fluorescent vedolizumab in intestinal biopsy samples. Patients with high numbers of detectable $\alpha 4 \beta 7$-expressing cells subsequently responded to vedolizumab, whereas patients with less detectable $\alpha 4 \beta 7$-expressing cells did not [7].

Molecular endoscopy represents a new approach to visualize in vivo signaling events in IBD patients and to develop a rational concept for the personalized use of biological agents in IBD. This may allow the selection of ideally suited neutralizing antibodies for the subsequent clinical therapy. Such targeted in vivo molecular endoscopy assists in optimizing the risk/benefit ratio of treatment in IBD patients.

\section{Conflict of Interest}

No conflict of interest has been declared by the author.

Author

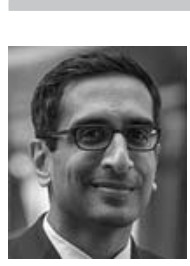

\section{Raja Atreya}

Prof. Dr., Medizinische Klinik I, Universitätsklinikum Erlangen, Deutschland

Correspondence

\section{Prof. Dr. Raja Atreya}

Medizinische Klinik I

Universitätsklinikum Erlangen

Ulmenweg 18

91054 Erlangen

Deutschland

raja.atreya@uk-erlangen.de

\section{References}

[1] Torres J, Mehandru S, Colombel JF et al. Crohn's disease. Lancet 2017; 389: 17411755

[2] Kobayashi T, Siegmund B, Le Berre C et al. Ulcerative colitis. Nat Rev Dis Primers 2020; 6: 74

[3] Atreya R, Neurath MF. Mechanisms of molecular resistance and predictors of response to biological therapy in inflammatory bowel disease. Lancet Gastroenterol Hepatol 2018; 3: $790-802$

[4] Digby-Bell JL, Atreya R, Monteleone G et al. Interrogating host immunity to predict treatment response in inflammatory bowe 
disease. Nat Rev Gastroenterol Hepatol 2020; 17: 9-20

[5] Atreya R, Zimmer M, Bartsch B et al. Antibodies gainst tumor necrosis factor (TNF) induce T-cell apoptosis in patients with inflammatory bowel diseases via TNF receptor 2 and intestinal CD14+ macrophages. Gastroenterology 2011; 141: 2026-2038

[6] Atreya R, Neumann H, Neufert C et al. In vivo imaging using fluorescent antibodies to tumor necrosis factor predicts therapeutic response in Crohn's disease. Nature Medicine 2014; 20: 313-318
[7] Rath T, Bojarski C, Neurath MF. Molecular imaging of mucosal $\alpha 4 \beta 7$ integrin expression with the fluorescent anti-adhesion antibody vedolizumab in Crohn's disease. Gastrointestinal Endoscopy 2017; 86: 406-408 